\title{
A pilot randomized controlled trial of a tailored smoking cessation program for people living with HIV in the Washington, D.C. metropolitan area
}

Elexis C. Kierstead ${ }^{1,2^{*}}$ D, Emily Harvey ${ }^{3}$, Denisse Sanchez ${ }^{1}$, Kimberly Horn ${ }^{4,5}$, Lorien C. Abroms ${ }^{6}$, Freya Spielberg ${ }^{7}$, Cassandra A. Stanton ${ }^{8}$, Charles Debnam ${ }^{9}$, Amy M. Cohn ${ }^{10}$, Tiffany Gray $^{11}$, Manya Magnus ${ }^{2}$, Minal Patel ${ }^{1}$, Raymond Niaura ${ }^{12}$ and Jessica L. Elf ${ }^{13}$

\begin{abstract}
Objective: Morbidity and mortality from smoking-related diseases among people living with HIV (PLWH) in the U.S. surpasses that due to HIV itself. Conventional smoking cessation treatments have not demonstrated strong efficacy among PLWH. We conducted a pilot randomized controlled trial (RCT) to evaluate a tailored smoking cessation intervention based on the minority stress model. We compared standard of care counseling (SOC) to a tailored intervention (TI) including one face-to-face counseling session incorporating cognitive behavioral therapy to build resilience, and 30 days of 2-way text messaging.

Results: The primary outcome was smoking cessation. Secondary outcomes included cigarettes per day (CPD), exhaled carbon monoxide (CO), and cessation self-efficacy. A total of 25 participants were enrolled (TI:11, SOC:14), and 2 were lost to follow-up. There were no significant differences in quit rates between study groups. However, there was a significantly greater decrease in CPD in the TI versus SOC (13.5 vs. 0.0, p-value:0.036). Additionally, self-efficacy increased in both groups (TI p-value:0.012, SOC p-value:0.049) and CO decreased in both groups (TI p-value: $<0.001$, SOC $p$-value:0.049). This intervention shows promise to support smoking cessation among PLWH. A larger study is needed to fully evaluate the efficacy of this approach.

Clinical trial: Trial Registration: Retrospectively registered (10/20/2020) NCT04594109.
\end{abstract}

Keywords: HIV/AIDS, Tobacco, Cessation, Behavioral health, Minority health

\section{Introduction}

Cigarette smoking prevalence among U.S. adults is currently $14 \%$ [1]. However, for people living with HIV (PLWH), smoking rates are 2 to 3 times higher, ranging from 40 to $60 \%$ [2-6]. In addition to a high smoking prevalence, PLWH are also at increased risk of negative health effects from smoking as compared to

*Correspondence: Ikierstead@truthinitiative.org

1 Schroeder Institute, Truth Initiative, 900 G St. NW, Washington, DC, USA

Full list of author information is available at the end of the article their seronegative peers [3, 6-11]. As PLWH are living longer due to advances in treatment, the morbidity and mortality attributed to smoking-related diseases among PLWH in the U.S. currently outweighs that due to HIV infection [12]. Importantly, more than two out of three PLWH who smoke cigarettes are interested in quitting $[4$, 13] however, standard smoking cessation strategies have shown only moderate efficacy [14].

Washington, D.C. (D.C.) has one of the highest rates of HIV in the U.S. (1.8\%) [15]. Additionally, among D.C. residents who are 50 or older, $3.2 \%$ are living with HIV 
[15]. The burden of disease falls most heavily on black men who have sex with men, as they make up the largest proportion of prevalent cases and newly diagnosed cases in D.C. [15]. According to data from a national sample, PLWH are at twice the risk of having an income at or below the poverty threshold [16]. Being a part of intersecting minority populations can lead to increased likelihood of mental health disorders and substance use, specifically tobacco $[15,17,18]$. A 2015 cross-sectional sampling of PLWH in D.C. found $40.6 \%$ identified as current smokers [19].

Prior research has described the ways in which stigma surrounding HIV has exacerbated smoking among PLWH [17], however limited research exists to combat smoking through specifically targeting these underlying determinants $[14,20]$. We developed and evaluated a tailored smoking cessation intervention for PLWH in the D.C. metropolitan area that integrates aspects of minority stress theory [18] utilizing counseling, pharmacotherapy, and mobile health (mHealth) methodologies. An interim analysis of this work has been presented virtually at the Society for Behavioral Medicine's annual conference in 2020 [21, 22].

\section{Main text}

\section{Methods}

We evaluated a newly developed tailored smoking cessation program for PLWH in D.C. via a pilot randomized controlled trial. This study was approved and overseen by the Chesapeake Institutional Review Board (IRB), now Advarra. All participants provided written informed consent prior to study enrollment.

A convenience sample of smokers was recruited via flyers in clinics and community centers serving PLWH, and word of mouth from September to December of 2017. Follow-up continued through February of 2018. Given the nature of the pilot study, sample size was determined based on feasibility and cost considerations. Participants were eligible if they were 1) 18 years or older, 2) living in the D.C. metro area, 3) self-reported PLWH, 4) currently smoking cigarettes daily, 5) willing to set a quit date within 7 days of the first meeting, 6) engaged in primary care, and 7) equipped with a mobile phone that could receive SMS text messages. At baseline, smoking status was confirmed by a carbon monoxide $(\mathrm{CO})$ breath test where smoking was defined as a reading of greater than or equal to seven parts per million (ppm) using the coVita Smokerlyzer [23]. Participants were excluded if they were 1) using smokeless tobacco or electronic cigarettes at least once per day, 2) currently enrolled in a quit smoking program, 3) using nicotine replacement therapy (NRT), 4) currently in an alcohol treatment program, 5) pregnant, breastfeeding or planning to become pregnant, or 6) diagnosed with heart disease or high blood pressure that was not controlled by medication. They were also excluded if they had a heart attack in the last 2 weeks, serious underlying irregular heartbeat, serious or worsening chest pain, or active TMJ syndrome.

Once determined eligible and consented, participants were randomly assigned 1:1 to either the intervention or control arm. A computer-generated randomization sequence was used for individual randomization. Neither the participant nor study staff were blinded. Outcomes were determined at a follow-up visit one month later. The trial was completed at the conclusion of the funding period.

\section{Study conditions}

Control Condition Participants were provided a one-time standard of care (SOC) in-person cessation counseling session lasting approximately one hour and received a 30-day supply of nicotine replacement therapy (NRT) consisting of nicotine gum and patches. The SOC cessation counseling was adapted from the current clinical practice guidelines [24].

Experimental Condition Participants in the intervention arm were provided a one-time tailored cognitivebehavioral therapy (CBT) in-person cessation counseling intervention (TI) lasting approximately one hour, a 30-day supply of NRT, and a tailored bi-directional text messaging program delivering two messages per day for four weeks. Participants were asked to bring their mobile phones and were instructed by study staff how to initiatie the text message program during their baseline visit. Two participants in the TI group were not enrolled due to mobile-phone difficulties at baseline. The TI session was adapted from the clinical practice guidelines to include the elements of our conceptual framework rooted in the minority stress model $[18,24]$. The intervention used a CBT approach to address issues of stress related to HIV stigma, minority status and socioeconomic condition. The conceptual framework supporting the TI theorizes that resilience-based coping has the potential to attenuate the negative effect of stigma on the relationship between stress and smoking. Positive coping strategies delivered to participants through the intervention are theorized to improve self-efficacy to cease smoking in the face of stress, adapting Teti et al.s work on resilience [25-28].

\section{Measures}

At the baseline session, information was collected on sociodemographic characteristics, smoking patterns, past cessation attempts, and perceived access to quitting resources.

Our primary outcome of interest was self-reported smoking cessation at 30-days after baseline, verified by a 
$\mathrm{CO}$ reading of less than seven ppm. Secondary outcomes included changes in cigarettes per day (CPD), CO levels, and self-efficacy for cigarette abstinence [29].

Self-efficacy for cigarette abstinence was evaluated using a validated tool from Spek et al. wherein participants were asked to rate their confidence in not smoking given certain situations [29]. Response options included a five-point scale ranging from, "certainly not" to "certainly," and the tool contained 6 items. Values $0-4$ were assigned to the response options for each item and values were averaged to create a summary score.

\section{Statistical analyses}

Participant characteristics and demographic variables obtained at baseline were compared across treatment groups using Pearson's Chi Squared or Fisher's Exact tests, as appropriate, for categorical variables. Wilcoxon Mann-Whitney tests were used to evaluate differences between the study arms for continuous variables. Frequencies and percentages were reported for categorical variables while medians and inter-quartile ranges (IQRs) were reported for continuous variables. To assess the primary outcome of smoking cessation, cessation status at follow-up was compared across treatment groups and Fisher's exact test was performed to assess differences between treatment arms.

An intent-to-treat (ITT) analysis was used wherein participants lost to follow-up $(n=2)$ were left in the dataset and were treated as if they did not quit and as if their continuous outcomes had not changed from baseline to follow-up. Scales for secondary outcomes were summarized by median value and change from baseline to follow-up. Change in scale from baseline to follow-up was evaluated across treatment groups using Wilcoxon Signed Rank Sum tests. All analyses were conducted using SAS 9.4 [30].

\section{Results}

\section{Participant characteristics}

Forty people were screened for the study and $n=25$ were enrolled, with two participants lost to follow-up. Those screened and not enrolled either did not meet eligibility criteria or did not attend their scheduled session. Eleven were randomized to the TI $(44 \%)$ and fourteen to the SOC (56\%) (Fig. 1). The median age of participants was 54 (IQR: 48,58 ). The majority were male $(\mathrm{n}=18 ; 72 \%)$, Black or African American $(\mathrm{n}=19$; $76 \%)$, not Hispanic $(n=24 ; 96 \%)$, unemployed $(n=16$; $64 \%)$, and making less than $\$ 20,000$ a year $(n=19$; 76\%). Approximately half identified as a sexual minority $(\mathrm{n}=13 ; 52 \%)$. The median age at which the participants were diagnosed with HIV was 32 (IQR: 25,43 ) and the median amount of time the participants had lived with

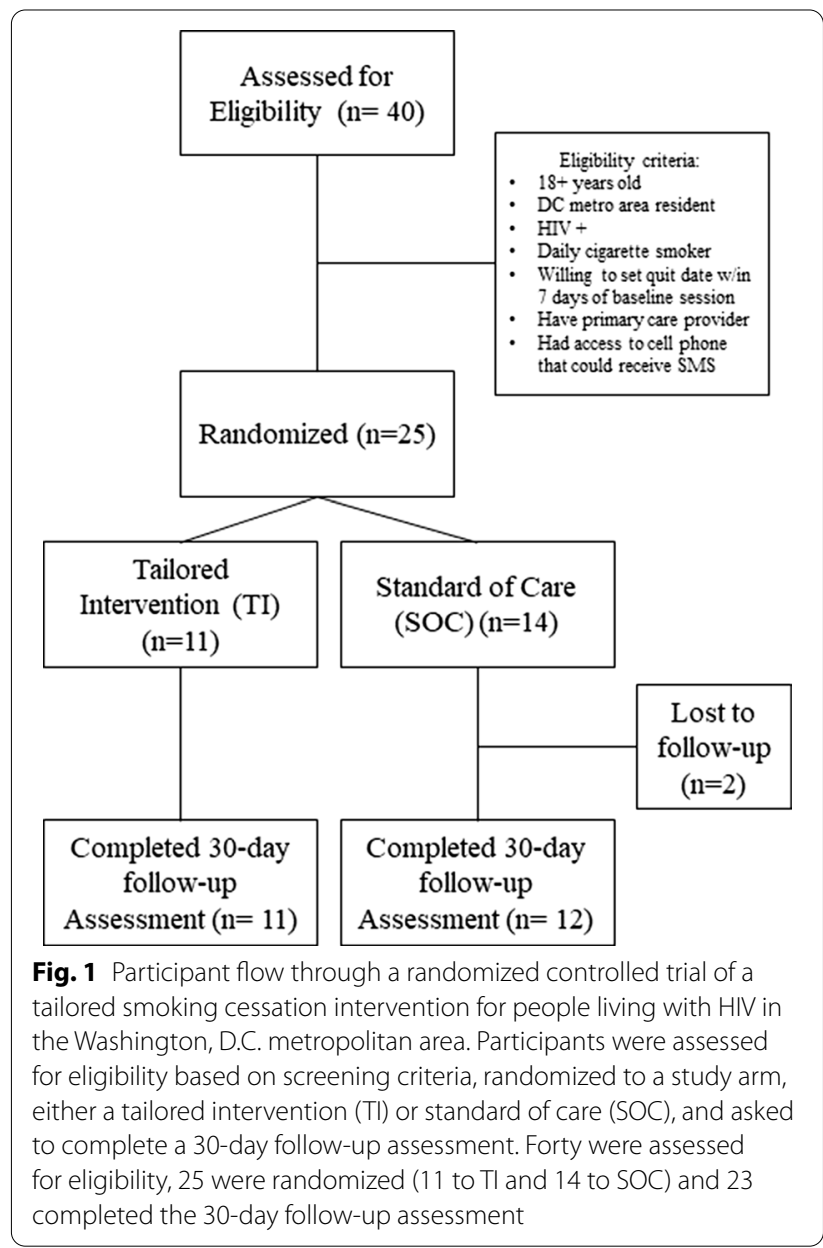

HIV was 19.5 years (IQR: 15, 28.5). There were no statistically significant differences between study arms (Table 1).

At baseline, participants smoked a median of 10 (IQR: $3,20)$ cigarettes per day. In the prior 12 months, approximately half of participants had tried to quit smoking $(\mathrm{n}=13 ; 52 \%)$. The median age at which participants had first smoked regularly was 18 (IQR: 16, 20). Most reported that they had not been advised to quit by a healthcare provider in the past 12 months $(n=18 ; 72 \%)$. Thirty percent did not know if quit smoking services are available at their place of HIV care and $17 \%$ reported that these services are not available (Table 1).

Nine participants $(82 \%)$ interacted with the text messaging program beyond initiating the program at the baseline session. Participants received a median of 71.5 text messages (IQR: 61,75) and sent a median of 8 text messages (IQR: 3, 10). Participants sent a minimum of 2 and a maximum of 22 text messages throughout the study. Most participants self-reportedly used the NRT provided $(\mathrm{n}=21 ; 84 \%)$. 
Table 1 Demographic characteristics of participants enrolled in a randomized controlled trial of a tailored smoking cessation intervention for people with HIV in the Washington, D.C. metro area, by treatment group $(n=25)$

\begin{tabular}{|c|c|c|c|c|}
\hline & $\begin{array}{l}\text { Total }(n=25) \\
\text { Median (IQR) }\end{array}$ & $\begin{array}{l}\text { Control }(n=14) \\
\text { Median (IQR) }\end{array}$ & $\begin{array}{l}\text { Intervention }(\mathrm{n}=11) \\
\text { Median (IQR) }\end{array}$ & P-value* \\
\hline Age & $54(48,58)$ & $51(47,57)$ & $58(51,60)$ & 0.0715 \\
\hline Cigarettes per day at baseline & $10(3,20)$ & $9(1,15)$ & $15(6,20)$ & 0.1224 \\
\hline Age first smoke regularly & $18(16,20)$ & $18.5(16,23)$ & $16(15,19)$ & 0.2173 \\
\hline Years smoking regularly & $37(27,41)$ & $32(23,38)$ & $38(35,44)$ & 0.0507 \\
\hline Age at HIV diagnosis & $32(25,43)$ & $31(25,37)$ & $32(24,44)$ & 0.4025 \\
\hline \multirow[t]{2}{*}{ Years living with HIV } & $19.5(15,28.5)$ & $19(15,27)$ & $20(13,28)$ & 0.9085 \\
\hline & $\mathrm{n}(\%)$ & n (\%) & n (\%) & \\
\hline Gender identity & & & & 0.8037 \\
\hline Male & $18(72)$ & $11(79)$ & $7(64)$ & \\
\hline Female & $5(20)$ & $2(14)$ & $3(27)$ & \\
\hline Other/Transgender & $2(8)$ & $1(7)$ & $1(9)$ & \\
\hline Race & & & & 0.1804 \\
\hline Not Black or African American & $6(24)$ & $5(36)$ & $1(9)$ & \\
\hline White & $1(4)$ & $1(7)$ & $0(0)$ & \\
\hline American Indian or Alaska Native & $1(4)$ & $1(7)$ & $0(0)$ & \\
\hline More than one race & $4(16)$ & $3(21)$ & $1(7)$ & \\
\hline Black or African American & $19(76)$ & $9(64)$ & $10(91)$ & \\
\hline Ethnicity & & & & 0.4400 \\
\hline Not of Hispanic, Latino or Spanish origin & $24(96)$ & $14(100)$ & $10(91)$ & \\
\hline Of Hispanic, Latino or Spanish origin & $1(4)$ & $0(0)$ & $1(9)$ & \\
\hline Sexual Identity & & & & 1.000 \\
\hline Straight/heterosexual & $12(48)$ & $7(50)$ & $5(45)$ & \\
\hline Sexual minority & $13(52)$ & $7(50)$ & $6(55)$ & \\
\hline Gay or lesbian/homosexual & $9(36)$ & $6(43)$ & $3(27)$ & \\
\hline Bisexual & $3(12)$ & $0(0)$ & $3(27)$ & \\
\hline Something else & $1(4)$ & $1(7)$ & $0(0)$ & \\
\hline Education & & & & 1.000 \\
\hline Some College or more & $15(60)$ & $8(57)$ & $7(64)$ & \\
\hline High School/GED/Vocational or Less & $10(40)$ & $6(43)$ & $4(34)$ & \\
\hline Employment & & & & 0.2077 \\
\hline Employed & $9(36)$ & $7(50)$ & $2(18)$ & \\
\hline Not Employed & $16(64)$ & $7(50)$ & $9(82)$ & \\
\hline Financial needs & & & & 0.4347 \\
\hline Meeting needs and more & $11(44)$ & $5(36)$ & $6(55)$ & \\
\hline Just/Not Meeting needs & $14(56)$ & $9(64)$ & $5(45)$ & \\
\hline Household income per year & & & & 0.6036 \\
\hline$\geq \$ 20,000$ & $4(16)$ & $3(23)$ & $1(10)$ & \\
\hline$<\$ 20,000$ & $19(76)$ & $10(77)$ & $9(90)$ & \\
\hline Prefer not to say & $2(8)$ & $1(7)$ & $1(9)$ & \\
\hline Menthol cigarette smoking & & & & 0.2300 \\
\hline Non-menthol & $3(12)$ & $3(21)$ & $0(0)$ & \\
\hline Menthol & $21(84)$ & $11(79)$ & $10(91)$ & \\
\hline No usual brand & $1(4)$ & $0(0)$ & $1(9)$ & \\
\hline Used of NRT during the study period & $21(84)$ & $11(79)$ & $10(91)$ & 0.6043 \\
\hline Tried to quit smoking in the past 12 months & $13(52)$ & $8(57)$ & $5(45)$ & 0.6951 \\
\hline $\begin{array}{l}\text { Were advised to quit smoking in the past } 12 \text { months by } \\
\text { a doctor or healthcare provider }\end{array}$ & $7(28)$ & $4(29)$ & $3(27)$ & 1.000 \\
\hline
\end{tabular}

Race and sexual minority categories were condensed for statistical testing due to small sample size, however the frequency and percentages of the condensed categories are shown in italics

${ }^{*} \mathrm{p}$-value comparing treatment arms 
Table 2 Primary and secondary outcomes of participants enrolled in a randomized controlled trial of a tailored smoking cessation intervention for people with HIV in the Washington, D.C. metro area, from baseline to 30-day follow-up, by treatment group $(n=25)$

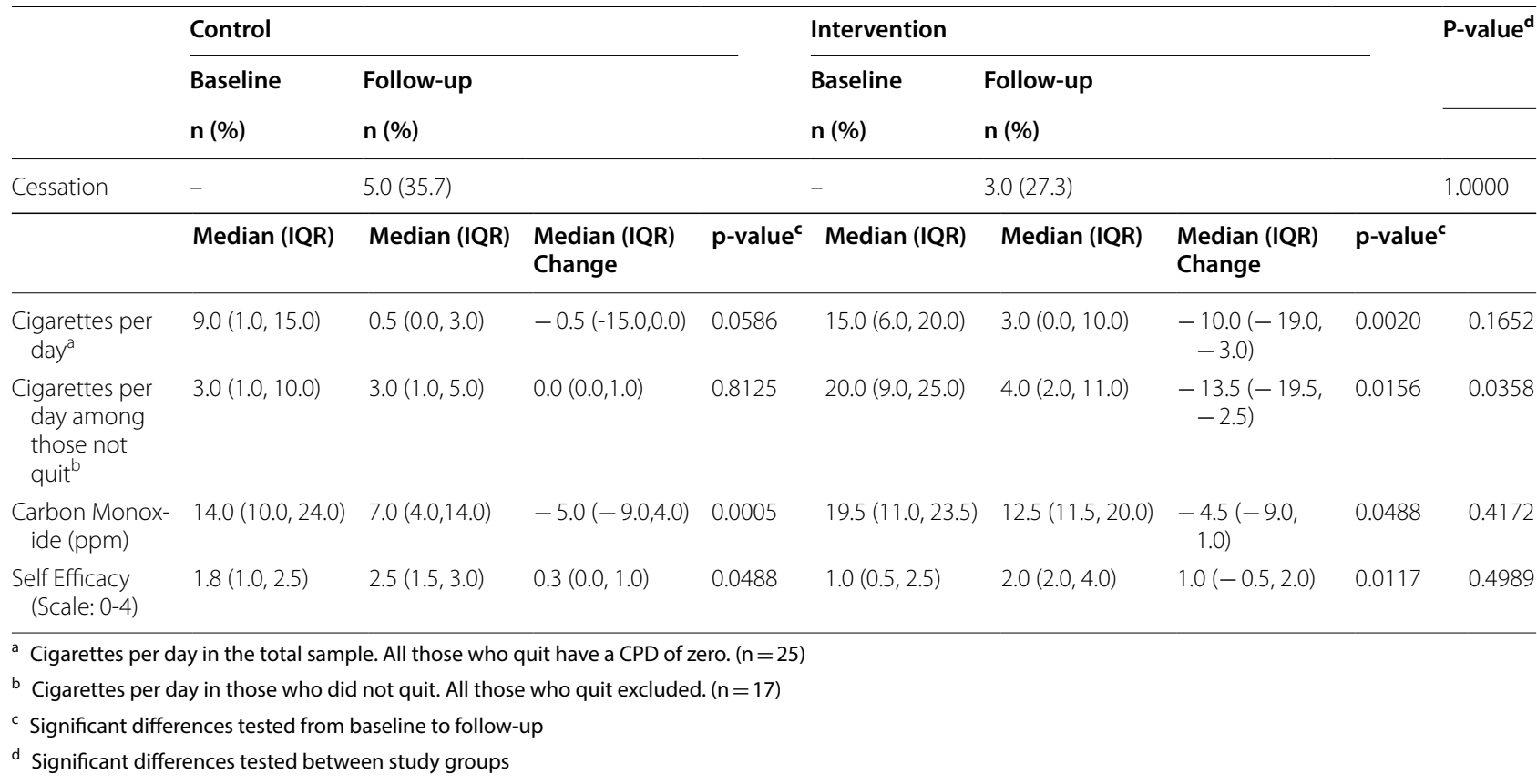

\section{Quitting outcomes}

In total, eight participants quit smoking by follow-up (32\%); this included five participants in the SOC group (35.7\%) and three participants in the TI group (27.3\%). All participants who self-reported that they had quit had $\mathrm{CO}$ levels below $7 \mathrm{ppm}$, verifying their status. Among those who did not quit, those in the TI group experienced significantly greater decreases in CPD than the SOC group (13.5 vs. $0.0, p=0.0358)$. There were significant differences between baseline and follow-up $\mathrm{CO}$ in both treatment groups (TI $\mathrm{p}=0.0488$, SOC $\mathrm{p}=0.0005$ ). Additionally, both the groups experienced significant increases in self-efficacy from baseline to follow-up (TI $\mathrm{p}=0.0117$, SOC $\mathrm{p}=0.0488)$ (Table 2).

\section{Discussion}

Our pilot project of a tailored smoking cessation program for PLWH in the Washington, D.C. metropolitan area showed promising results. The majority of participants in the intervention group were actively engaged in the text messaging program. Both the TI and SOC groups had 30 -day cessation rates at around $30 \%$. The intervention arm, however, saw significantly greater reductions in CPD among those who did not quit than the control arm. Both arms demonstrated significant decreases in $\mathrm{CO}$ and significant increases in self-efficacy to abstain from smoking from baseline to follow-up. These data were analyzed ITT. Although there was variation in significance and magnitude, the overall trends did not change when the sample was analyzed "as-treated."

Although participants receiving the intervention in this small study were not more likely to quit than those who received SOC, it is important to note that $32 \%$ of the total sample $(\mathrm{n}=8)$ did successfully quit smoking at one month. According to the CDC, less than 1 in 10 smokers have quit successfully in the past year despite approximately half of all smokers reportedly making a quit attempt [31]. Additionally, in a comparable cessation intervention for PLWH that also had a 30-day follow-up among a larger sample of 95 smokers living with HIV, Vidrine et al. evaluated a cell phone delivered counseling intervention and found that those in the study quit smoking at a rate of $8 \%$ in the control group and $21 \%$ in the intervention group; lower quit rates than what can be seen in this study. Although this sample did not see a significant difference between the intervention and control groups, the rates at which participants quit smoking after only 30-days was on par with previous research [32].

Additionally, although the control group received a "standard of care" cessation counseling treatment, it is unlikely this is truly the standard of what PLWH are experiencing in their regular clinical care as few participants reported having been advised to quit by their healthcare providers in the prior year. Although PLWH are consistently engaged with the healthcare system, providers may be missing opportunities to intervene 
and may not be providing standard of care counseling at the level necessary to create change.

These data suggest that this tailored intervention could be a promising strategy for smoking cessation among PLWH. Though it was not shown to be superior to a standard of care intervention in this small study for complete cessation, the intervention did significantly decrease number of cigarettes smoked, and positively impacted changes in self-efficacy, a key activating component of the conceptual model. Larger studies providing greater exposure to the counseling program are needed to fully evaluate the efficacy of this intervention.

\section{Limitations}

- The sample was small, reducing the ability to account for possible cofounders via adjustment.

- Short follow-up time.

- Secondary outcomes were self-reported, not accounting for some biases.

- Due to enrollment errors, two of eleven participants in the treatment group did not receive the full text messaging program.

\section{Abbreviations}

PLWH: People living with HIV; RCT: Randomized controlled trial; D.C: Washington D.C; SOC: Standard of care; CBT: Cognitive behavioral therapy; TI: Tailored intervention; NRT: Nicotine replacement therapy; CO: Carbon monoxide.

\section{Acknowledgements}

The authors would like to thank the participants for generously volunteering their time. The authors would also like to acknowledge the contributions of Barbara Schillo (Truth Initiative ${ }^{\circledR}$ ) for her review and input on the manuscript.

\section{Authors' contributions}

JE and RN were the principal investigators. JE, RN, KH, CS, CD, AC, TG, FS, and LA conceptualized the study. JE, RN, DS, EK and EH developed the study materials and protocols. CD facilitated participant recruitment. EK and EH collected the data. EK analyzed the data with guidance from MM and JE. LK, $\mathrm{JE}, \mathrm{MM}$ and MP contributed to manuscript development. All authors reviewed manuscript drafts and contributed feedback. All authors read and approved the final manuscript.

\section{Funding}

This research was funded by the DC Metro Tobacco Research and Instruction Consortium (MeTRIC) from The Milken Institute School of Public Health at The George Washington University, Washington DC and was supported by Truth Initiative ${ }^{\circledR}$, Washington DC. This research has been facilitated by the services provided by the District of Columbia Center for AIDS Research, an NIH funded program (Al1 17970), which is supported by the following $\mathrm{NIH} \mathrm{Co-Funding} \mathrm{and}$ Participating Institutes and Centers: NIAID, NCI, NICHD, NHLBI, NIDA, NIMH, NIA, FIC, NIGMS, NIDDK, and OAR. The content is solely the responsibility of the authors and does not necessarily represent the official views of the NIH.

\section{Availability of data and materials}

This data is housed at Truth Initiative and is not publicly available.

\section{Ethics approval and consent to participate}

This study was approved and overseen by the Chesapeake Institutional Review Board (IRB), now Advarra (Pro00022163). All participants provided written informed consent prior to study enrollment.

\section{Consent for publication}

Not applicable.

\section{Competing interests}

One author has potential conflicts of interests to declare. Dr. Manya Magnus (GW) has received grants from the NIH for HIV Prevention Trials Network Clinical Research site and for the DC Center for AIDS, although she has received no funds directly. She is also the author of three epidemiology textbooks through Jones and Barlett Inc. from which she receives royalties.

\section{Author details}

${ }^{1}$ Schroeder Institute, Truth Initiative, 900 G St. NW, Washington, DC, USA. ${ }^{2}$ Department of Epidemiology, Milken Institute School of Public Health, The George Washington University, Washington, DC, USA. ${ }^{3}$ The Henry M. Jackson Foundation for the Advancement of Military Medicine, Bethesda, MD, USA.

${ }^{4}$ Carilion Fralin Biomedical Research Institute at VTC, Virginia Polytechnic Institute and State University, Blacksburg, VA, USA. ${ }^{5}$ Department of Population Health Sciences, Virginia-Maryland College of Veterinary Medicine, Virginia Polytechnic Institute and State University, Blacksburg, VA, USA. ${ }^{6}$ Department of Prevention and Community Health, Milken Institute School of Public Health, The George Washington University, Washington, USA. ${ }^{7}$ Department of Population Health, Dell Medical School, University of Texas, Austin, TX, USA. ${ }^{8}$ Behavioral Health and Health Policy Practice, Westat, Rockville, MD, USA. ${ }^{9}$ Community Wellness Alliance, Washington, DC, USA. ${ }^{10}$ Department of Pediatrics, University of Oklahoma College of Medicine, Oklahoma City, OK, USA. ${ }^{11}$ Department of Community Health Administration, Department of Health, Washington, DC, USA. ${ }^{12}$ Department of Social and Behavioral Sciences, School of Global Public Health, New York University, New York City, NY, USA. ${ }^{13}$ Department of Environmental and Radiological Health Sciences, College of Veterinary Medicine \& Biomedical Sciences, Fort Collins, CO, USA.

Received: 30 July 2020 Accepted: 9 December 2020

Published online: 06 January 2021

\section{References}

1. Wang TW, Asman K, Gentzke AS, Cullen KA, Holder-Hayes E, Reyes-Guzman C, et al. Tobacco Product Use Among Adults — United States, 2017. CDC; 2018 November 9, 2018. Contract No.: 44.

2. Browning KK, Wewers ME, Ferketich AK, Diaz P. Tobacco use and cessation in HIV-infected individuals. Clin Chest Med. 2013;34(2):181-90.

3. Lifson AR, Lando HA. Smoking and HIV: prevalence, Health Risks, and Cessation Strategies. Current HIV/AIDS Rep. 2012;9(3):223-30.

4. Mamary EM, Bahrs D, Martinez S. Cigarette smoking and the desire to quit among individuals living with HIV. AIDS Patient Care STDs. 2002;16(1):39-42.

5. Mdodo R, Frazier EL, Dube SR, Mattson CL, Sutton MY, Brooks JT, et al. Cigarette smoking prevalence among adults with HIV compared with the general adult population in the United States: cross-sectional surveys. Ann Intern Med. 2015;162(5):335-44.

6. Niaura R, Shadel WG, Morrow K, Tashima K, Flanigan T, Abrams DB. Human immunodeficiency virus infection, AIDS, and smoking cessation: the time is now. Clin Infect Dis Official Public Infect Dis Soc Am. 2000;31(3):808-12.

7. Pacek $L R$, Cioe PA. tobacco use, use disorders, and smoking cessation interventions in persons living with HIV. Current HIV/AIDS Rep. 2015;12(4):413-20.

8. Pacek LR, Harrell PT, Martins SS. Cigarette smoking and drug use among a nationally representative sample of HIV-positive individuals. Am J Addictions/American Academy of Psychiatrists Alcoholism Addictions. 2014;23(6):582-90.

9. Miguez-Burbano MJ, Burbano X, Ashkin D, Pitchenik A, Allan R, Pineda L, et al. Impact of tobacco use on the development of opportunistic respiratory infections in HIV seropositive patients on antiretroviral therapy. Addiction Biol. 2003;8(1):39-43. 
10. Phillips AN, Neaton J, Lundgren JD. The role of HIV in serious diseases other than AIDS. AIDS. 2008;22(18):2409-18.

11. Mocroft A, Reiss P, Gasiorowski J, Ledergerber B, Kowalska J, Chiesi $A$, et al. Serious fatal and nonfatal non-AIDS-defining illnesses in Europe. Journal of acquired immune deficiency syndromes (1999). 2010;55(2):262-70.

12. Helleberg M, Afzal S, Kronborg G, Larsen CS, Pedersen G, Pedersen C, et al. Mortality attributable to smoking among HIV-1-infected individuals: a nationwide, population-based cohort study. Clin Infect Dis Official Publication Infect Dis Soc Am. 2013;56(5):727-34.

13. Tesoriero JM, Gieryic SM, Carrascal A, Lavigne HE, Behavior. Smoking Among HIV Positive New Yorkers: Prevalence, Frequency, and Opportunities for Cessation. J AIDS. 2010;14(4):824-35.

14. Ledgerwood DM, Yskes R. Smoking cessation for people living with HIV/AIDS: a literature review and synthesis. J Nicotine Tobacco Res. 2016;18(12):2177-84.

15. District of Columbia Department of Health. Annual Epidemiology \& Surveillance Report: Data Through December 2018. Washington, DC: HIV/ AIDS, Hepatitis, STD and TB Administration (HAHSTA),; 2019.

16. Denning P, DiNenno E. Communities in Crisis: Is There a Generalized HIV Epidemic in Impoverished Urban Areas of the United States? 2017.

17. Crockett KB, Rice WS, Turan B. Associations Between Multiple Forms of Discrimination and Tobacco Use Among People Living With HIV: The Mediating Role of Avoidance Coping. Journal of acquired immune deficiency syndromes (1999). 2018;78(1):9-15.

18. Meyer IH. Prejudice, social stress, and mental health in lesbian, gay, and bisexual populations: conceptual issues and research evidence. Psychol Bull. 2003;129(5):674-97.

19. Levy M, Greenberg A, Hart R, Powers Happ L, Hadigan C, Castel A, et al. High burden of metabolic comorbidities in a citywide cohort of HIV outpatients: evolving health care needs of people aging with HIV in Washington DC. HIV Med. 2017;18(10):724-35.

20. Moscou-Jackson G, Commodore-Mensah Y, Farley J, DiGiacomo M. Smoking-Cessation Interventions in People Living With HIV Infection: a Systematic Review. J Assoc Nurses AIDS Care. 2014;25(1):32-45.

21. Kierstead EC, Harvey E, Sanchez D, Horn K, Abroms LC, Spielberg F, et al. 2020 ABM Annual Meeting Abstracts Supplement. In: Revenson TA, Ruiz JM editors. Annals of Behavioral Medicine2020. p. S1-S827.
22. Kierstead EC, Harvey E, Sanchez D, Horn K, Abroms LC, Spielberg F, et al. editors. A pilot randomized controlled trial of a tailored smoking cessation program for people living with HIV in the Washington, DC metropolitan area. Society of Behavioral Medicine Annual Meeting; 2020 May 2020; Virtual.

23. CoVita. Smokerlyzer 2020 [Available from: https://www.covita.net/thesmokerlyzer-range/.

24. A Clinical Practice Guideline for Treating Tobacco Use and Dependence: 2008 Update: A U.S. Public Health Service Report. American Journal of Preventive Medicine. 2008;35(2):158-76.

25. Earnshaw VA, Lang SM, Lippitt M, Jin H, Chaudoir SR. HIV stigma and physical health symptoms: do social support, adaptive coping, and/or identity centrality act as resilience resources? AIDS Behavior. 2015:19(1):41-9.

26. Livingston NA, Christianson N, Cochran BN. Minority stress, psychological distress, and alcohol misuse among sexual minority young adults: a resiliency-based conditional process analysis. Addict Behav. 2016;63:125-31.

27. Marlatt GA, Witkiewitz K. Relapse prevention for alcohol and drug problems. 2005;2:1-44

28. Teti M, Martin AE, Ranade R, Massie J, Malebranche DJ, Tschann JM, et al. "I'ma Keep Rising. I'ma Keep Going Forward, Regardless" Exploring Black Men's Resilience Amid Sociostructural Challenges and Stressors. Qualitative health research. 2012;22(4):524-33.

29. Spek V, Lemmens F, Chatrou M, van Kempen S, Pouwer F, Pop V. Development of a smoking abstinence self-efficacy questionnaire. Int J Behav Med. 2013;20(3):444-9.

30. SAS Institute Inc. SAS 9.4. Cary, NC: SAS Institute Inc.; 2013.

31. Babb S. Quitting smoking among adults_-United States, 2000-2015. Morbidity mortality weekly report. 2017;65.

32. Vidrine DJ, Arduino RC, Lazev AB, Gritz ER. A randomized trial of a proactive cellular telephone intervention for smokers living with HIV/AIDS. 2006;20(2):253-60.

\section{Publisher's Note}

Springer Nature remains neutral with regard to jurisdictional claims in published maps and institutional affiliations.
Ready to submit your research? Choose BMC and benefit from:

- fast, convenient online submission

- thorough peer review by experienced researchers in your field

- rapid publication on acceptance

- support for research data, including large and complex data types

- gold Open Access which fosters wider collaboration and increased citations

- maximum visibility for your research: over 100M website views per year

At $B M C$, research is always in progress.

Learn more biomedcentral.com/submissions 